\title{
Study of Synthetic Airspeed Algorithm Based on Machine Learning for Lift Coefficient Curve Fitting
}

\author{
Dianzhong Chen ${ }^{1}$, Yue $\mathrm{Xu}^{2}$, Lei Wang ${ }^{3}$ \\ \{dc2e12@163.com¹, xuyue2@comac.cc²,wanglei13@comac.cc $\left.{ }^{3}\right\}$
}

COMAC Shanghai Aircraft Design \& Research Institute, No. 5188, Jinke Road, Shanghai, China ${ }^{1,2,3}$

\begin{abstract}
Traditional air data system of airplane utilizes pitot probe for airspeed measurement. However, problems such as icing and bird strike will lead to failure of pitot probe. Airspeed display loss is rated as disastrous loss status. Airspeed calculation algorithm based on inertial data and movable surface positions (status of flaps and slats) has been studied by the Boeing Company and the Airbus Company and applied in airplane models of Boeing 787 and Airbus A350. Commercial Airplane of China has been dedicated in studying algorithm of airspeed calculation. Study indicates the importance of accurate lift coefficient identification for different flight configurations under certain attack angles. Theoretical analysis indicates the relationship of piecewise linearity between lift coefficient and attack angle. Based on the above relationship, machine learning algorithm of support vector regression (SVR) is applied to process air data. Furthermore, synthetic airspeed algorithm is proposed and verified.
\end{abstract}

Keywords: synthetic airspeed algorithm, support vector regression (SVR).

\section{Introduction}

Airspeed is an important air data provided in air data system (ADS) [1]. ADS, a multiple input multiple output (MIMO) system, measures air data of total pressure, static pressure, attack angle, sideslip angle, total temperature through sensors of pitot probe, static port, attack angle sensor, total temperature sensor, and provides data for subsystems, such as flight control system, flight management system, fuel system [2,3]. Accurate airspeed data is the basis for operation of flight control system [4,5]. However, accidents such as icing and bird strike will cause failure of pitot probe.

To avoid disastrous loss status of airspeed display loss, airspeed calculation algorithm without total pressure data is studied, which can provide a non similar data source, as a supervision for pitot probe state and a substitute in case of pitot probe system (two or three pitot probes with voting algorithm) failure. Chen proposes an airspeed calculation method based on inertial navigation data and wind field data [6]. While, wind field changes rapidly and accuracy of data from weather forecast is not high enough. A method for Mach number and true airspeed calculation using data of altitude from global positioning system (GPS) and 
inertial reference system (IRS) is put forward in patent [7]. However, it is known that neither of these two systems can give accurate altitude data with enough precision, for problems of positioning algorithm and accumulative error, respectively. Airspeed calculation through data from inertial navigation system (INS) and flight control system (FCS) exhibits acceptable results, reported in [8] and [9]. Up till now, mature airspeed calculation is only applied at advanced airplane models of Boeing 787 and Airbus A350.

In the paper, a synthetic airspeed algorithm based on INS and FCS data is to be proposed. To deal with nonlinear characteristic of attack angle-lift coefficient curve, machine learning algorithm of SVR is to be applied. Air data from two flight tests will be used to verify the accuracy of the proposed synthetic airspeed algorithm.

\section{Synthetic Airspeed Modeling}

Synthetic airspeed calculation relies on Expression (1) between impact pressure $q_{c}$ and calibrated airspeed $V_{C A S}$,

$$
V_{C A S}=C_{S 0}\left(5\left[\left(q_{c} / P_{S 0}+1\right)^{7 / 2}-1\right]\right)^{1 / 2}
$$

in which $C_{S O}$ is the conversion ratio from Mach to Knot, $P_{S O}$ is the normal atmosphere pressure, $q_{c}$ is the impact pressure, denoting actual pressure sensed by a moving creature. According to Expression (1), synthetic calibrated airspeed $V_{C A S}$ is the function of parameter impact pressure $q_{c}$. When airspeed is smaller than $0.3 \mathrm{M}$, impact pressure $q_{c}$ is approximately equal to dynamic pressure $q_{b a r}$, which is defined under the condition of impressible fluid, calculated by:

$$
q_{c} \approx q_{b a r}=q_{t}-q_{s}
$$

where $q_{t}$ and $q_{s}$ are total pressure and static pressure, respectively. Total pressure $q_{t}$ and static pressure $q_{s}$ can be sensed by pitot probes and static port sensors. While, when airspeed is larger than $0.3 \mathrm{M}$, air is compressible, and impact pressure is unequal to dynamic pressure. Mach number will reflect the compressibility of air. The difference between $q_{c}$ and $q_{b a r}$ increase with the rise in Mach number. However, a more accurate $q_{c}$ could be obtained through correction on $q_{b a r}$ by Mach.

Except for operation between $q_{t}$ and $q_{s}$, dynamic pressure $q_{b a r}$ can be derived through the following equation: 


$$
q_{b a r}=\frac{m N_{z}}{S C_{L}}
$$

in which $m, N_{z}, S, C_{L}$ respect gross weight, load factor (Z-axis acceleration), reference wing area, lift coefficient, respectively. The above four parameters on the right of Equation (3) are real-time values. Gross weight change of an airplane mainly comes from fuel consumption and is monitored by flight management computer. Z-axis acceleration is sensed by inertial navigation module. Movable surface positions (status of flaps and slats) influence reference wing area (parameter $S$ ) and lift coefficient $C_{L}$ simultaneously. In the following study, given the multiplication relationship between $S, C_{L}$, and relatively small area change of $S$ caused by different movable surface positions, $S$ is approximately regarded as a constant value. Influence by movable surface area is reflected in the value of $C_{L}$. Patent $[10]$ reveals that $C_{L}$ is modeled as:

$$
C_{L}=C_{L 0}+\Delta C_{L}+C_{L \alpha} * \alpha,
$$

in which $C_{L O}$ is the lift coefficient with movable surfaces at 0 position when angle of attack $\alpha$ equals to $0, \Delta C_{L}$ is the lift coefficient increase caused by movable surfaces, $C_{L \alpha}$ is the derivative of lift coefficient $C_{L}$ to angle of attack $\alpha$.

For the designed airplane (Model XXX), finite element analysis is conducted to study the relationship between lift coefficient $C_{L}$ and attack angle $\alpha$. Simulation result (Figure 1) reveals characteristics of nonlinearity and piecewise linearity. Based on the characteristics, machine learning method of Support Vector Machine (SVM) is to be studied to fit the curve.

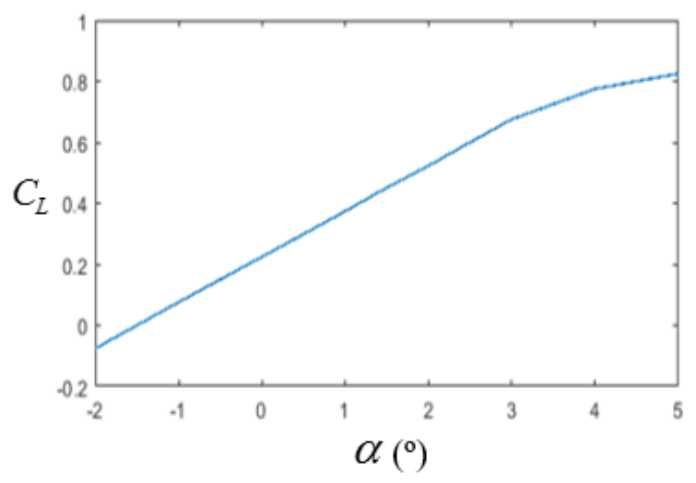

Fig. 1. Lift coefficient $C_{L}$ when attack angle $\alpha$ changes from $-2^{\circ}$ to $5^{\circ}$. 


\section{Piecewise Fitting Based on Support Vector Regression}

Common nonlinear fitting methods include cubic polynomial fit, least square fit, nonlinear approximation based-on neural network. SVM, a machine learning method developed from statistical learning theory, is widely applied in model fitting of sensor outputs [11,12]. SVM improves generalization capacity through structural risk minimization principle, solving problems such as curse of dimensionality, small-sample learning [13,14]. Advantage of SVM compared with other fitting methods is that SVM outputs globally optimal solution with good generalization capacity [15].

For the lift coefficient fitting problem as Figure 1, piecewise linearity function fitting is a simple solution, with fast computation speed. However, high fitting precision cannot be achieved. For methods such as polynomial fit and neural network fit, piecewise linearity may lead to the problem of overfitting. Therefore, machine learning method of multiple support vector regression machine (MSVRM) is selected as a promising method.

Fitting principle is shown as Figure 2, in which $X_{i}$ is the input vector of the ith support vector regression machine (SVRM), and $Y_{i}$ is the output of the ith SVRM [16]. According to the lift coefficient curve of Figure 1, attack angle input space is segmented into subspaces of $X_{1}, X_{2}, \cdots X_{n}$. Input space cover the whole input range of attack angles and subspaces do not intersect between each other. A SVRM is constructed for each subspace according to the relationship between attack angle and lift coefficient. All SVRMs have the same structure but with different parameters. For each SVRM, training samples are collected at corresponding subspace, and SVRM is trained independently to approach actual output characteristic of the subspace. After constructing SVRMs, the final OUTPUT Y $\left(C_{L}\right)$ at the entire input range $(\alpha)$ is obtained.

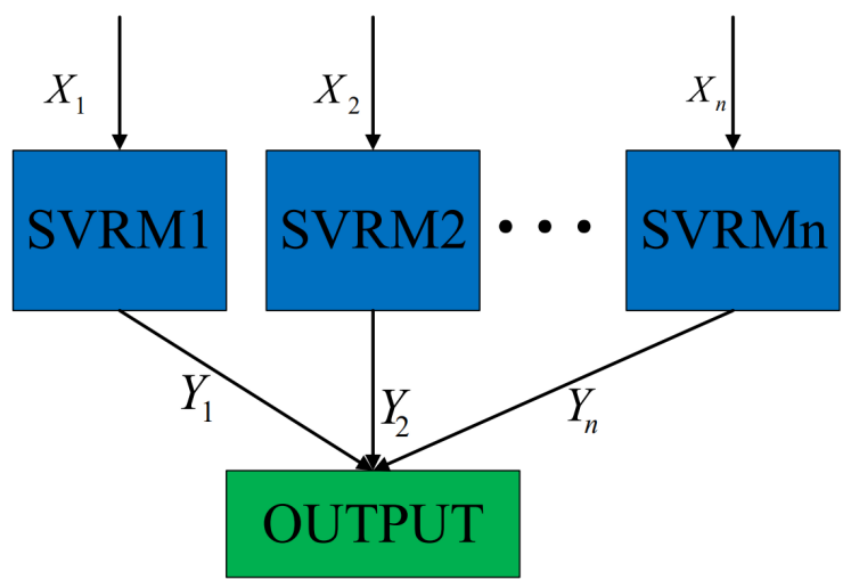

Fig. 2. Principle of fitting based-on MSVRM. 
According to the theory of statistical learning, SVM is to look for the optimum separating hyperplane satisfying classification requirements, which results in the maximum distance between samples nearest to the separating hyperplane. In most cases, sample set is linearly indivisible, and a kernel function is selected to map the original function for nonlinear transformation. Nonlinear transformation $\phi: x \rightarrow \varphi(x)$ transforms the given samples to certain high-dimensional feature space. Thus, separating hyperplane is constructed in highdimensional feature space. A separating hyperplane can be expressed in original space as:

$$
\omega \sqcap \varphi(x)+b=0
$$

in which $\omega, \varphi(x)$ are n-dimension vectors, $b$ is the threshold, $(\sqcup)$ respects inner product.

The problem of looking for optimum separating hyperplane can be transferred to a problem of convex quadratic optimization:

$$
\min \left(\frac{1}{2}\|\omega\|^{2}+c \sum_{i=1}^{n} \xi\right)
$$

which satisfies constraint condition:

$$
y_{i}\left(\omega \bullet \varphi\left(x_{i}\right)+b\right) \geq 1-\xi i=1,2, \cdots, n \text {, }
$$

in which $c$ is the penalty coefficient and $\xi$ is the relaxation factor.

Penalty coefficient $c$ reflects the degree of compromise between classification precision and model complexity. The larger $c$ is, the higher fitting degree is, however, leading to higher complexity of SVM and the problem of overfitting. On the contrary, if $c$ is too small, punishment to empirical error is not enough, leading to SVM of low complexity but the problem of underfitting. For the problem of lift coefficient curve fitting, penalty coefficient $c$ is selected from an index series $\left[2^{-12}, 2^{11}\right]$. Experiments reveal that, if $c$ is larger than $2^{9}$, increase of $c$ has little effect on the performance of SVM, while complexity of SVM model puts too much load on computer. Cross validation tests are conducted for $c$ value selection. According to test results (Table 1), when penalty coefficient $c$ is confirmed as $2^{5}$, accuracy of cross validation $(83 \%)$ is the highest. 
Table 1. Penalty coefficient $\mathrm{c}$ and corresponding accuracy of cross validation.

\begin{tabular}{cc}
\hline Penalty coefficient $c$ & Accuracy of cross validation \\
\hline $2^{-12}$ & $62.3 \%$ \\
$2^{-11}$ & $68.5 \%$ \\
$2^{1}$ & $71.2 \%$ \\
$2^{2}$ & $73.6 \%$ \\
$2^{3}$ & $78.1 \%$ \\
$2^{4}$ & $81.1 \%$ \\
$2^{5}$ & $83.0 \%$ \\
$2^{5.5}$ & $81.2 \%$ \\
$2^{6}$ & $79.3 \%$ \\
$2^{9}$ & $79.5 \%$ \\
$2^{10}$ & $79.6 \%$ \\
$2^{11}$ & $79.6 \%$ \\
\hline
\end{tabular}

To improve SVM calculating speed and the algorithm stability, an improved SVM basedon least square method is proposed. Based on structural risk minimization principle, solving of $\omega$ and $b$ comes down to minimizing:

$$
\min \left(\frac{1}{2}\|\omega\|^{2}+c \sqsubset \varepsilon_{e}\right),
$$

in which $\varepsilon_{e}$ represents loss function.

Loss function is defined as: 


$$
\varepsilon_{e}=\frac{1}{2 k} \sum_{i=1}^{k} \lambda_{i} e_{i}^{2}
$$

in which $k, e_{i}, \lambda_{i}$ are sample size, error, independent weighing coefficient, respectively.

Independent weighing coefficient $\lambda_{i}$ is applied for different sample point, which means a major weighing coefficient is adopted for the sample point with minor nonlinear error. Then, the optimization problem is transferred to:

$$
\min J(\omega, b, e)=\frac{1}{2}\|\omega\|^{2}+\frac{c}{2 k} \sum_{i=1}^{k} \lambda_{i} e_{i}^{2},
$$

satisfying the condition:

$$
y_{i}\left[\omega\lceil\varphi(x)+b]=1-e_{i} .\right.
$$

Lagrange function is applied to resolve the above constrained extreme value problem:

$$
L(w, b, e, a)=J(w, b, e)-\sum_{i=1}^{k} \alpha_{i}\left\{y_{i}\left[\omega \llbracket \varphi\left(x_{i}\right)+b\right]-1+e_{i}\right\}
$$

in which $\alpha_{i}(\mathrm{i}=1,2, \cdots k)$ represents Lagrange multiplier, $e$ is the vector expressed as $\left(e_{1}, e_{2}, \cdots, e_{k}\right)$.

The fitting model finally obtained to conduct curve fitting is:

$$
f(x)=\sum_{i=1}^{l}\left(\alpha_{i}^{*}-a_{i}\right) k\left(x_{i}, x\right)+b,
$$

in which kernel function $k\left(x_{i}, x\right)$ is to replace mapping of inner product. 
Proper choice of kernel function is the precondition of correct recognition, which is conducted as: a number of common kernel functions such as polynormal kernel, Gaussian kernel, RBF kernel, Fourier kernel, are selected and SVM of different types are constructed; fitting effects in terms of calculating speed, fitting precision are compared; finally, Fourier kernel is selected to be the kernel function of MSVRM for the fitting of the attack angle-lift coefficient curve.

Based on the study of MSVRM above, piecewise fitting is conducted as the following procedures:

(1) Match values of attack angle with corresponding lift coefficient as sample couples;

(2) Divide the attack angle-lift coefficient curve into linear regions;

(3) According to the region division result, divide sample space into corresponding subspaces and train 100 sample couples in each subspace respectively;

(4) For each subspace, based-on different kernel functions, model identification is conducted, and parameters of $\alpha_{i}, b$ are confirmed;

(5) To compare SVRM fitting performance with different kernel functions, another 100 sample couples are tested to compare fitting results. Results reveal that Fourier kernel outputs the best fitting result.

\section{Synthetic Airspeed Calculation Based on Impact Pressure}

Airspeed synthetization based on air data (without using air pressure data) is conducted as the following procedures:

(1) Transform air data of real time fuel quality, $\mathrm{Z}$ axis acceleration, from binary number to decimal number according to encoding rules of corresponding storages;

(2) On the basis of the study in Section 3, fit attack angle-lift coefficient curve based on SVRM. SVRM produces expressions of lift coefficient with attack angles as input parameter;

(3) The attack angle-lift coefficient curve is based-on the configuration with flaps and slats at 0 position (clean configuration). For different configurations during two flight tests (flight test A and flight test B), gains of lift coefficient compared with clean configuration are identified through data processing. The gain is also related to the difference between impact pressure and dynamic pressure as analyzed in Equation (2).

Obtaining of gains of lift coefficient for different configurations makes it possible for airspeed estimation based on Equations (1) (3), as a secondary airspeed source. Air data during flight test A with a high altitude of around 24000 feet (configuration 1), a low altitude of around 4000 feet (configuration 2) and flight test B with a mid-altitude of 150000 feet (configuration 3 ) are extracted and airspeed calculation is conducted based on the data. 
Concrete flap and slat positions for different configurations are not given here for sake of confidentiality.

Figure 3(a) and Figure 3(d) illustrate measured dynamic pressure and calculated dynamic pressure with gains of $\mathrm{k} 1$ (corresponding to configuration 1) and $\mathrm{k} 2$ (corresponding to configuration 2) in flight test A, respectively. Figure 3(b), 3(c), 3(e), 3(f) indicate that calculated airspeeds approximate measured airspeeds respectively at steady flight stages with errors within 10 knots. For it is a primary study of airspeed calculation, emphasis is put on steady flight stage (with small acceleration). Figure 3(g) reveals ascent and descending processes are sharp with large acceleration, which is to be researched further.

Figure 4(a) Figure 4(g) illustrate results of airspeed calculation for flight test B. In the test, airplane is steady at a mid altitude and with a relatively slow ascent process. Airspeed calculation is conducted in both processes. Gains for slow ascent process and steady process at the mid altitude are $\mathrm{k} 3$ and $\mathrm{k} 4$, respectively. An obvious error exists between time from $4000 \mathrm{~s}$ to $5200 \mathrm{~s}$. From flight management computer, it is known that attack angle adjustment and configuration variation are conducted during the period, which result in a dynamic lift coefficient $C_{L}$. Except for this period, airspeed calculation error is within 10 knots (impulse noises are not considered).

In conclusion, airspeed calculation to flight test $\mathrm{A}$ and flight test $\mathrm{B}$ as procedures $1 \sim 3$ outputs synthetic airspeeds, based on inputs from flight management computer (real-time fuel quality, configuration information), inertial navigation system (acceleration), with errors within 10 knots for steady flight stage and slow ascent stage.

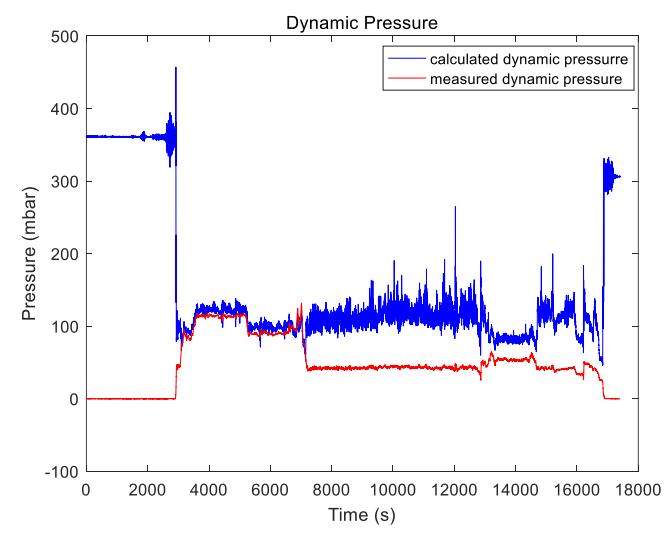

(a) Measured and calculated dynamic pressure with gain $\mathrm{k} 1$. 


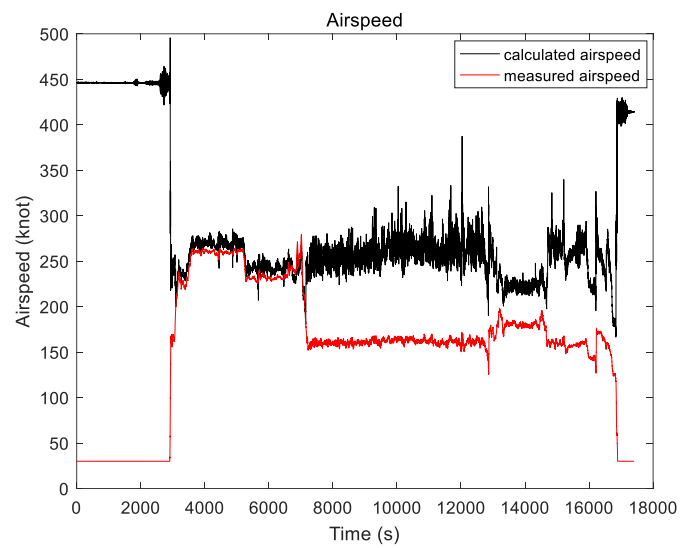

(b) Measured and calculated airspeed with gain k1.

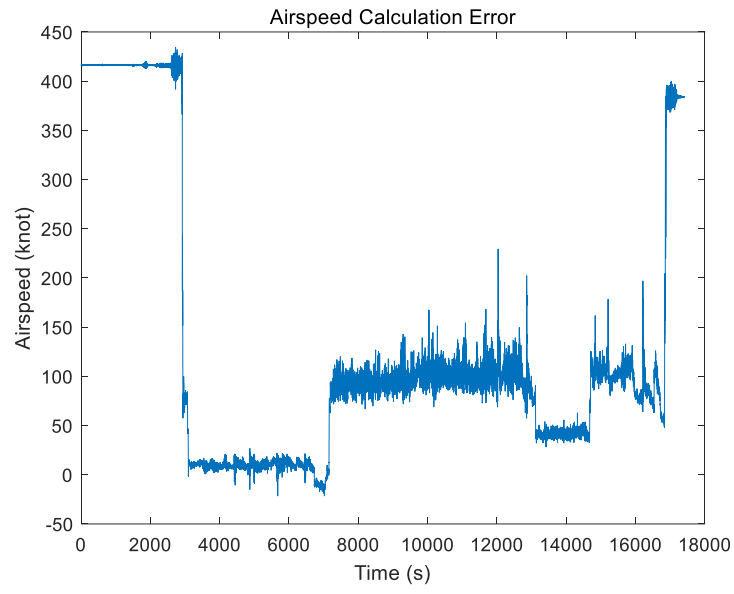

(c) Airspeed calculation error with gain k1. 


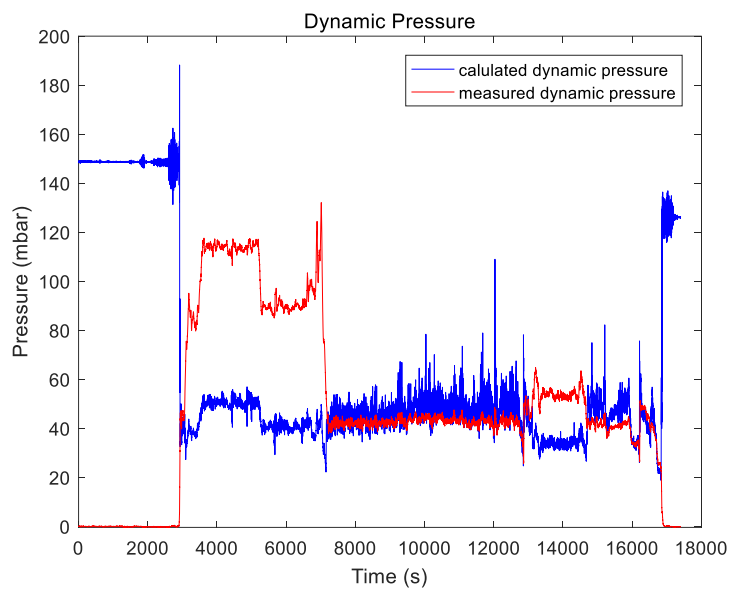

(d) Measured and calculated dynamic pressure with gain k2.

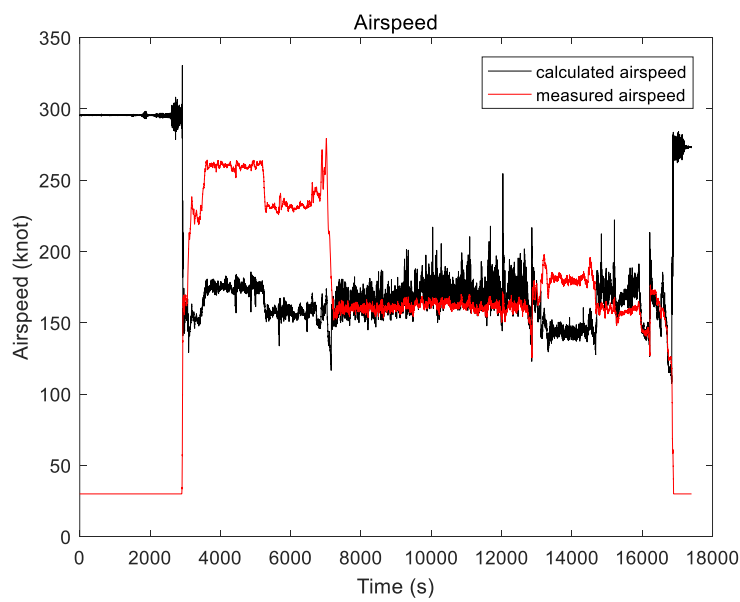

(e) Measured and calculated airspeed with gain k2. 


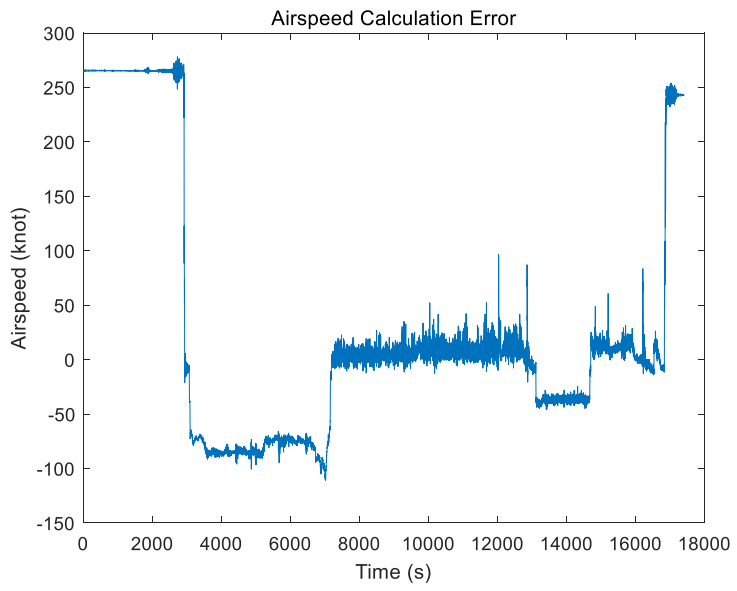

(f) Airspeed calculation error with gain k2.

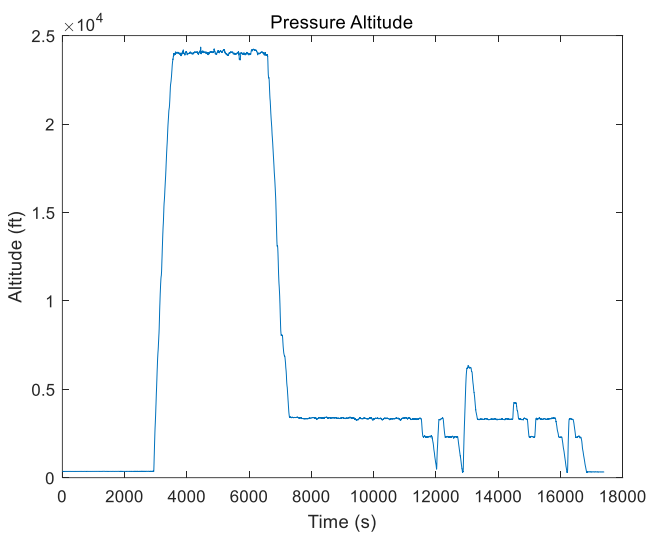

(g) Pressure altitude from traditional air data system. 
Fig. 3. Airspeed calculation during flight test A.

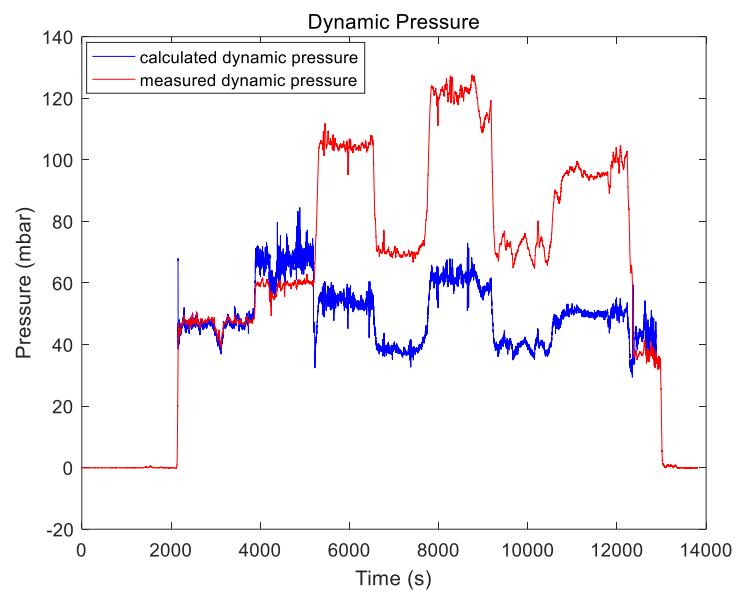

(a) Measured and calculated dynamic pressure with gain k3.

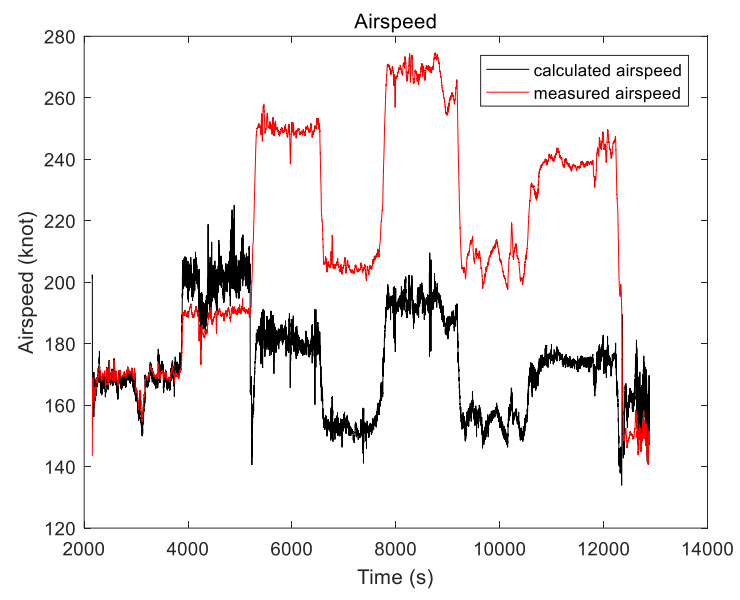

(b) Measured and calculated airspeed with gain k3. 


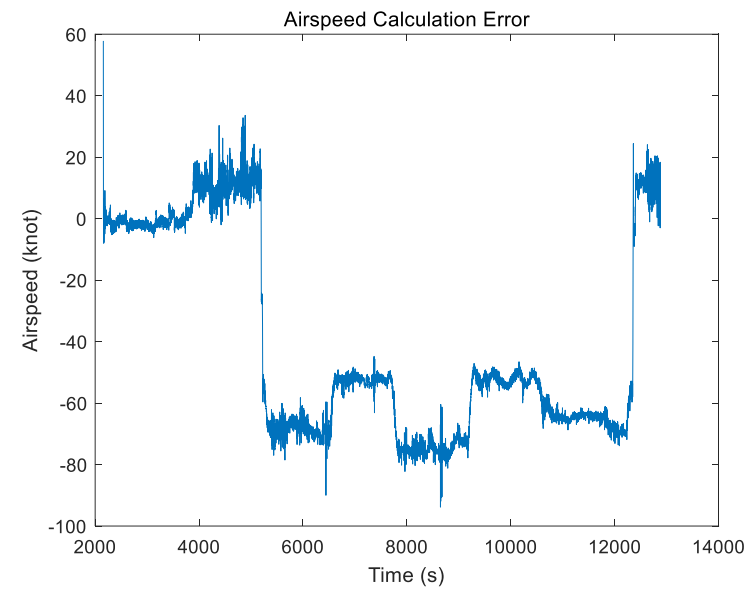

(c) Airspeed calculation error with gain $\mathrm{k} 3$.

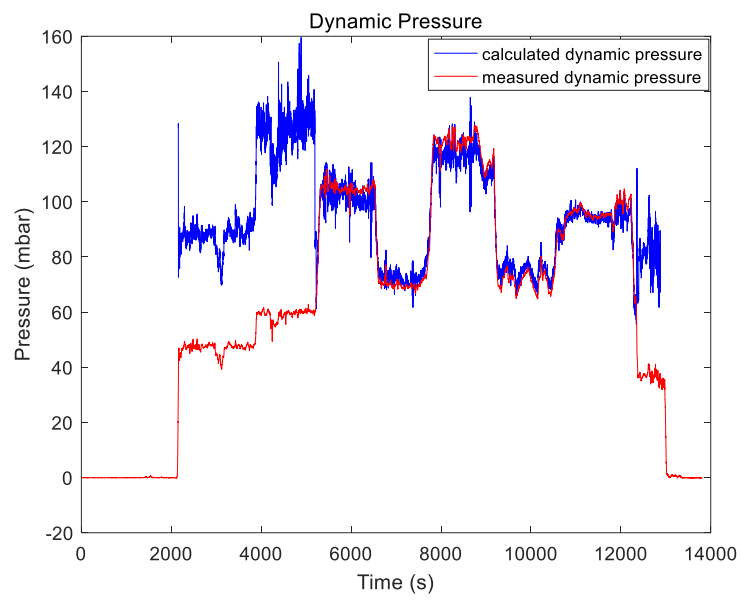

(d) Measured and calculated dynamic pressure with gain k4. 


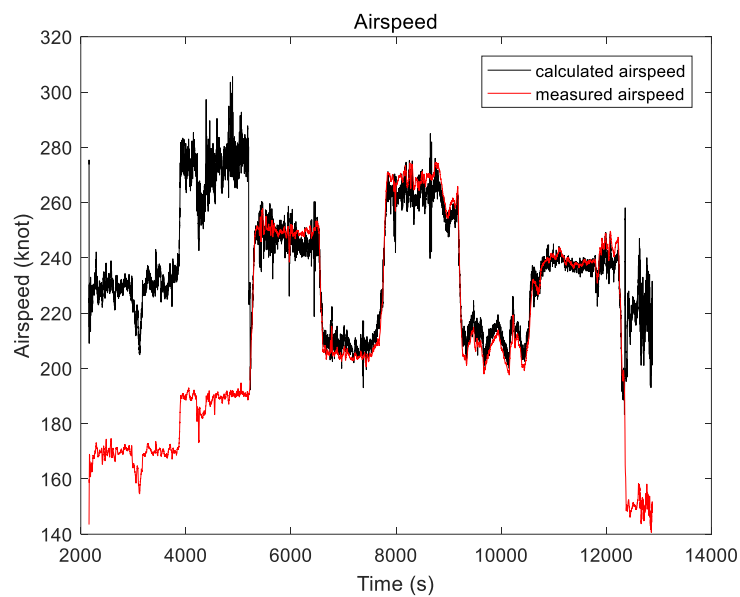

(e) Measured and calculated airspeed with gain $\mathrm{k} 4$.

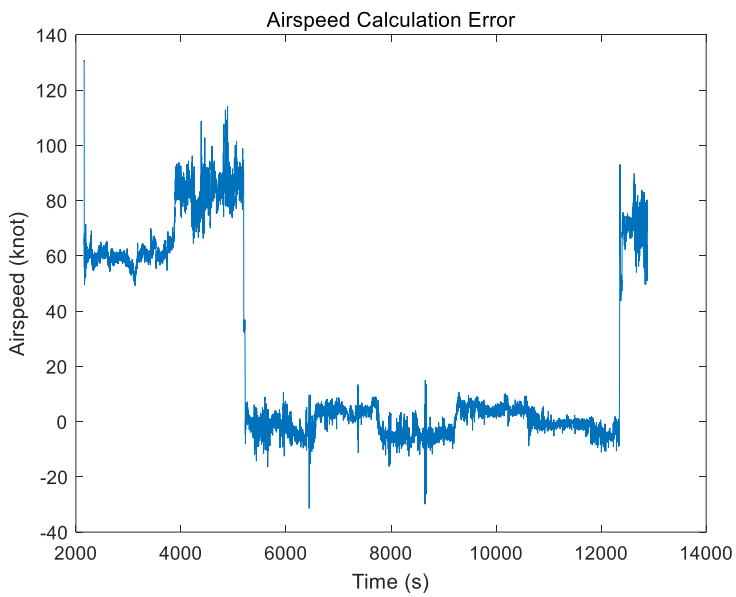

(f) Airspeed calculation error with gain k4. 


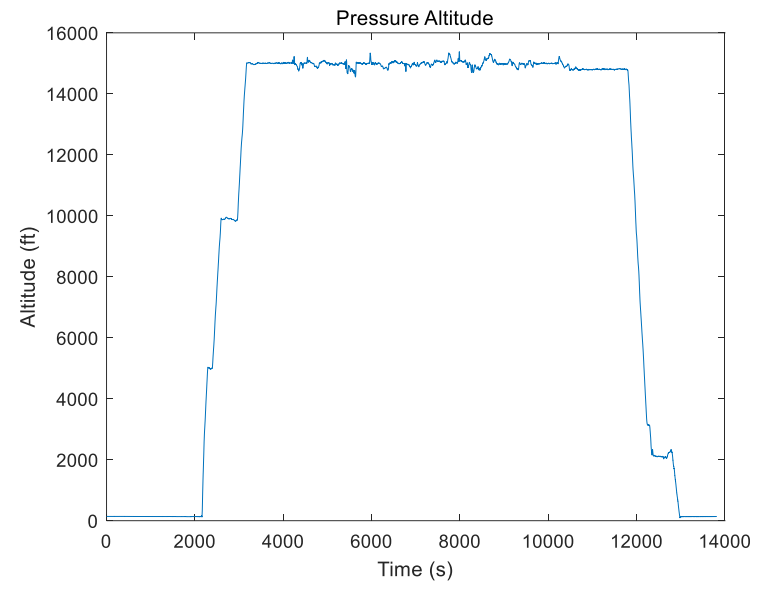

(g) Pressure altitude from traditional air data system.

Fig. 4. Airspeed calculation during flight test B.

\section{Conclusion}

An airspeed calculation algorithm is proposed in the paper. To deal with the problem of attack angle-lift coefficient curve nonlinearity, machine learning algorithm of SVR is studied and applied. To verify the accuracy of the proposed algorithm, air data from two flight tests is utilized for airspeed calculation. Results reveal that for steady flight stage and slow ascent stage, errors are within 10 knots. Further study will focus on airspeed calculation of steady flight stages in other configurations and motion stages with large accelerations. Moreover, other machine learning algorithms will be studied to deal with the problem of attack angle-lift coefficient curve nonlinearity. 


\section{References}

[1] Li Q D, Chen L L, Zhang X G: Flush airdata sensing system fast intelligent fault detection and diagnosis technology. Systems Engineering and Electronics, 31(10), pp. 2544-2546 (2009).

[2] Kai Chen, Jie Yan, Panfeng Huang: Design of Air Data System of an Aircraft Integrated Electronic Standby Instrument. Proceedings of the 2006 IEEE International Conference on Mechatronics and Automation, Luoyang China (June 25-28, 2006).

[3] Hyun Woo Roh, Yun Jin Park, Nam Eun Park, etal: Air Data System Calibration of T-50/A-50. AIAA Atmospheric Flight Mechanics Conference and Exhibit, Keystone, Colorado (Aug 21-24, 2006).

[4] Vivek Yadav, Radhakant Padhi, and S. N. Balakrishnan: Robust/Optimal Temperature Profile Control of a High-Speed Aerospace Vehicle Using Neural Networks. IEEE TRANS-ACTIONS ON NEURAL NETWORKS, 18(4), pp. 1115-1128 (2007).

[5] F.Adhika Pradipta Lie, Demoz Gebre-Egziabher: Synthetic Air Data System. Journal of Aircraft, 50(4), pp. 1234-1249 (2013).

[6] Lu Chen, Li Rongbing, etc: Calculation Method for Air Data Based on Information from Inertial Navigation System and Wind Field. Proceedings of 2016 IEEE Chinese Guidance, Navigation and Control Conference, Nanjing, China (August 12-14, 2016).

[7] Nathan V T, Anandappan T: Determination method for calculating at least one of first true airspeed of first Mach number of aircraft in flight determines true airspeed of first Mach number from group consisting of e.g. standard atmospheric data, thrust data. (2015).

[8] Kevin A Wise: Flight Testing of the X-45A J-UCAS Com-putational Alpha-Beta System. Keystone, Colorado: AIAA Guidance, Navigation, and Control Conference, 2006.

[9] M.L. Fravolini, M. Pastorelli, S. Pagnottelli, P. Valigi: Model-Based approaches for the Airspeed Estimation and Fault Monitoring of an Unmanned Aerial Vehicle. IEEE Workshop on Environmental Energy \& Structural Monitoring Systems (EESMS), pp.18-23 (2012). 
[10] The Boeing Company: Alternative Method to Determine the Air Mass State of An Aircraft and to Validate and Augment the Primary Method. (May 20, 2014).

[11] Fu Jingqi, Li Jing: Multi-parameter Differential Pressure Flowmeter Nonlinear Calibration Based on SVM. Proceedings of 4th international conference on Intelligent Computing, pp. 896-903 (2008).

[12] Wang Xiaodong, Zhang Haoren: Nonlinear dynamic compensation of sensors based on least squares support vector machines. Journal of Test and Measurement Technology, 20 (2), pp. 184-188 (2006).

[13] Vapnik V, Golowich S, Smola A: Support vector method for function approximation, regression estimation and signal processing. Adv Neural Infor Proc Syst. pp. 281-287 (1997).

[14] Ma Junshui, Theiler J, Perkins: Accurate on-line support vector regression. Neural Computation, 15 (11), pp. 2683-2703 (2003).

[15] Vapnik V: The nature of statistical learning theory. Spring Verlag, New York (1999).

[16] Dianzhong Chen: Study on a Rotational Micro-gyroscope Based on Magnetic Self-restoring Effect and the Detection Method. Harbin, China: Harbin Institute of Technology (Feb, 2019). 